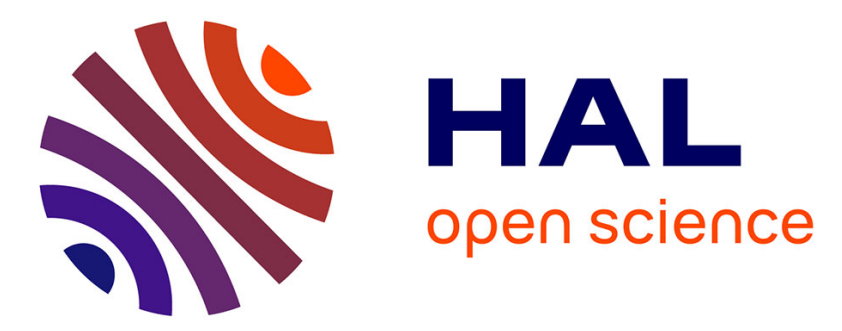

\title{
Quantum well infrared photo-detectors operating in the strong light-matter coupling regime
}

Pierre-Baptiste Vigneron, Stefano Pirotta, Iacopo Carusotto, Ngoc-Linh Tran, Giorgio Biasiol, Jean-Michel Manceau, Adel Bousseksou, Raffaele Colombelli

\section{- To cite this version:}

Pierre-Baptiste Vigneron, Stefano Pirotta, Iacopo Carusotto, Ngoc-Linh Tran, Giorgio Biasiol, et al.. Quantum well infrared photo-detectors operating in the strong light-matter coupling regime. Applied Physics Letters, 2019, 114 (13), pp.131104. 10.1063/1.5084112 . hal-02364795

\section{HAL Id: hal-02364795 https://hal.science/hal-02364795}

Submitted on 19 Nov 2020

HAL is a multi-disciplinary open access archive for the deposit and dissemination of scientific research documents, whether they are published or not. The documents may come from teaching and research institutions in France or abroad, or from public or private research centers.
L'archive ouverte pluridisciplinaire HAL, est destinée au dépôt et à la diffusion de documents scientifiques de niveau recherche, publiés ou non, émanant des établissements d'enseignement et de recherche français ou étrangers, des laboratoires publics ou privés. 


\section{Quantum well infrared photo-detectors operating in the strong light-matter coupling regime}

Cite as: Appl. Phys. Lett. 114, 131104 (2019); https://doi.org/10.1063/1.5084112

Submitted: 04 December 2018 . Accepted: 06 March 2019 . Published Online: 02 April 2019

Pierre-Baptiste Vigneron, Stefano Pirotta, lacopo Carusotto (D), Ngoc-Linh Tran (D), Giorgio Biasiol (D), Jean-Michel Manceau, Adel Bousseksou, and Raffaele Colombelli
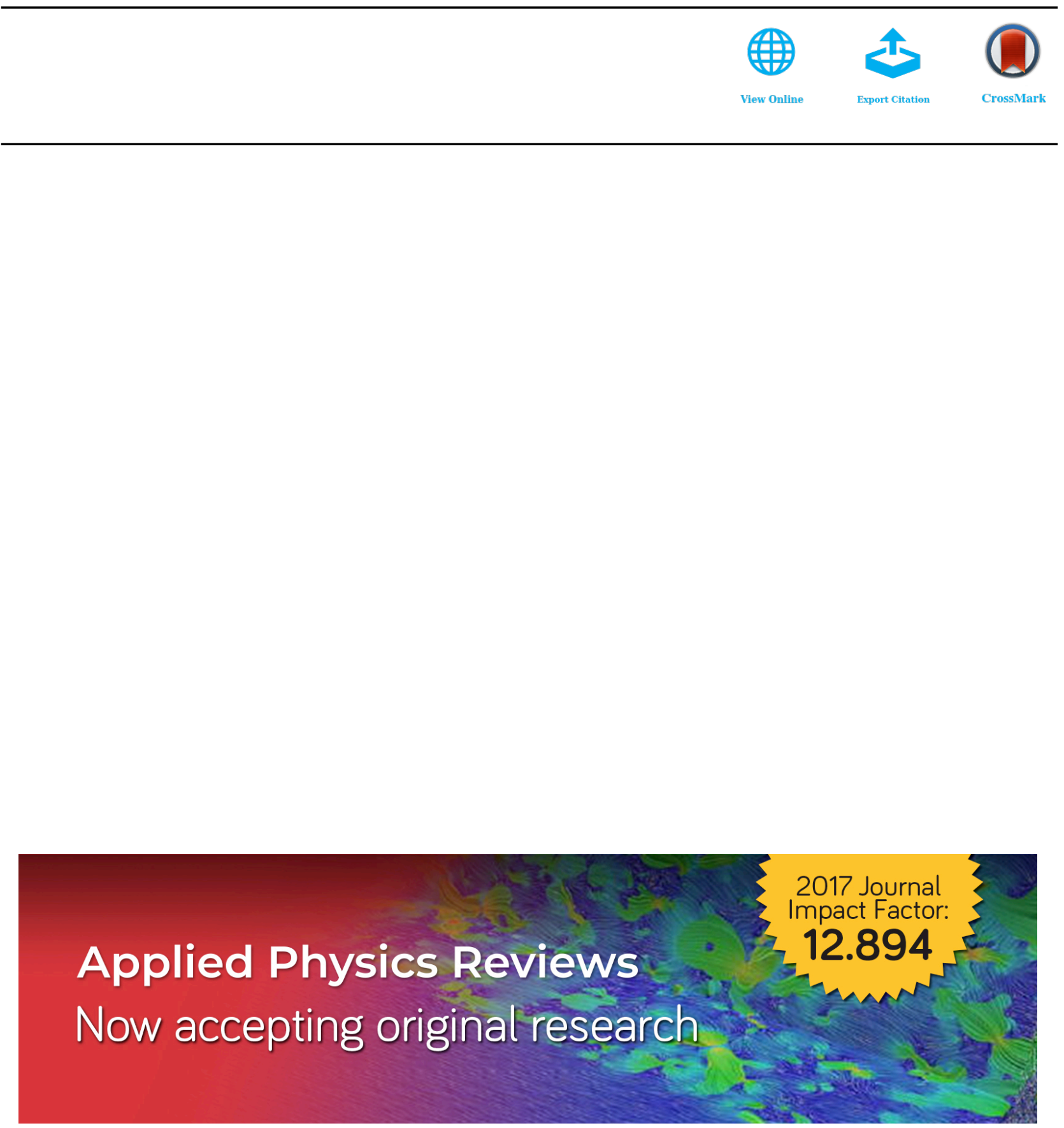


\title{
Quantum well infrared photo-detectors operating in the strong light-matter coupling regime
}

\author{
Cite as: Appl. Phys. Lett. 114, 131104 (2019); doi: 10.1063/1.5084112 \\ Submitted: 4 December 2018 • Accepted: 6 March 2019 . \\ Published Online: 2 April 2019
}

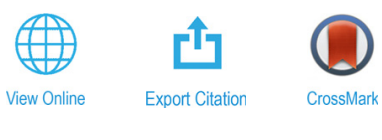

Pierre-Baptiste Vigneron, ${ }^{7}$ Stefano Pirotta, ${ }^{1}$ lacopo Carusotto, ${ }^{2}$ iD Ngoc-Linh Tran, ${ }^{1}$ (D) Giorgio Biasiol, Jean-Michel Manceau, ${ }^{7}$ Adel Bousseksou, ${ }^{7}$ and Raffaele Colombelli, ${ }^{1, a)}$

\author{
AFFILIATIONS \\ ${ }^{7}$ Centre de Nanosciences et de Nanotechnologies (C2N), CNRS UMR 9001, Université Paris-Sud, Université Paris-Saclay, 91120 \\ Palaiseau, France \\ ${ }^{2}$ Istituto Nazionale di Ottica, Bose-Einstein Condensation Center, University Trento, 38123 Trento, Italy \\ ${ }^{3}$ Laboratorio TASC, CNR-IOM, Area Science Park, S.S. 14 km 163.5, Basovizza, I-34149 Trieste, Italy \\ a)E-mail: raffaele.colombelli@u-psud.fr
}

\begin{abstract}
We report quantum well mid-infrared photo-detectors operating in the strong light-matter coupling regime. We claim that this is an ideal system to elucidate the elusive problem in intersubband polaritonics of the injection of electrons (single-particle fermionic states) into polaritonic modes (bosonic excitations). By reversing the perspective and focusing on the electron extraction, we have obtained experimental information on the transfer function between a polaritonic system and an electronic reservoir. In addition to its interest for fundamental science, this approach also opens promising avenues in view of adding previously unavailable functionalities to quantum well detectors and improving their performance.
\end{abstract}

Published under license by AIP Publishing. https://doi.org/10.1063/1.5084112

Infrared detectors operating in the mid-IR spectral range $(3 \mu \mathrm{m}-20 \mu \mathrm{m})$ are of crucial importance for a variety of applications, namely, spectroscopy, space observation, trace gas sensing, and imaging. One of the most prominent architectures is the quantum well infrared photodetector (QWIP), ${ }^{1}$ featuring high sensitivity and high speed, ${ }^{2}$ albeit with background limited operation (BLIP) only at very low temperatures, especially in the 2 nd atmospheric window $(8 \mu \mathrm{m}$ $<\lambda<12 \mu \mathrm{m}$ ). To date, these devices have mostly been operated in the weak light matter coupling regime. Operation in the strong lightmatter coupling regime [henceforth termed strongly coupled QWIP (SC-QWIP)] of intersubband (ISB) detectors would be interesting for both fundamental and applicative reasons.

There is current interest in developing LEDs (and lasers) based on ISB polaritons because they would have improved functionalities with respect to current sources, as detailed in Refs. 3-5. Efficient electrical injection of a polaritonic device, which would make them technologically interesting, is however a major challenge that is still unresolved today. The reason is that most of the electrons injected from an electronic reservoir into a polaritonic system tunnel into the dark states. ${ }^{6}$ This not only hampers the device efficiency but also makes it impossible to optically study the tunnel-coupling process, since the dark states do not couple to the electromagnetic field and are thus invisible. In order to get information on the microscopic mechanisms underlying the tunnel-coupling process, we suggest that the reverse process-i.e., the tunneling of a polariton (bosonic excitation) into an electronic (single particle) state-can be studied much more easily in a detector, because dark states do not play an important role as incoming photons only excite bright polariton modes. This relative simplicity of the detection process compared to the injection one is a strong motivation to develop SC-QWIPs and SC-QCDs (quantum cascade detectors) as a promising system to investigate the elusive interplay between optics and transport in strong light-matter coupled intersubband polaritonic devices. This is the main objective of this work.

An additional, applicative motivation, which is beyond the immediate scope of this paper, is related to the device BLIP operation (noise or temperature), which is essentially locked to the detection wavelength $\left[\mathrm{E}_{\mathrm{g} \rightarrow \text { e, }}\right.$, see Fig. $\left.1(\mathrm{a})\right]$ in a device operating in weak coupling regime. One strategy to increase the $\mathrm{T}_{\mathrm{BLIP}}$ has been developed in Ref. 2, by making the devices extremely small. As the effective capture area of a sub-wavelength-scale resonant dipole is approximately $\sim 3 \lambda^{2}$ / $8 \pi$ (see Ref. 7), reducing the surface leads to a reduction of the dark current that is larger than the reduction of the photocurrent. An alternative solution, which is the long term vista of this work, is to operate 
(a)

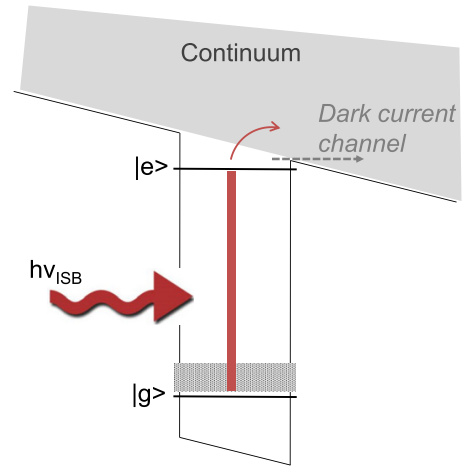

(b)

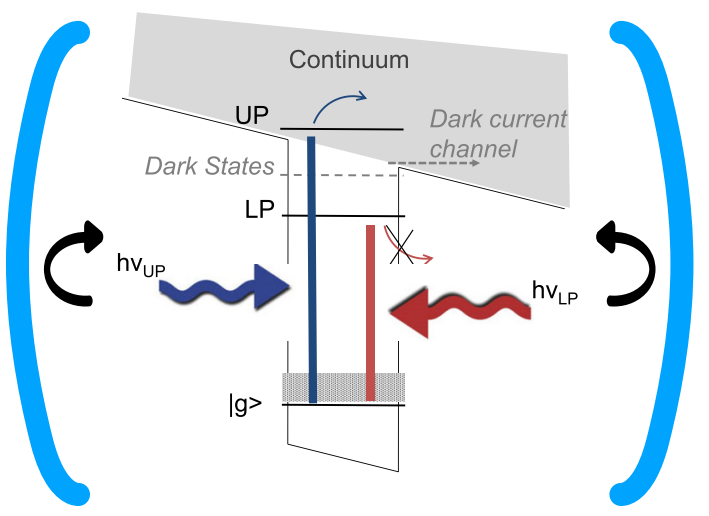

FIG. 1. (a) Schematics of the electronic levels of a quantum well infrared photodetector operating in weak coupling. The position of the excited level $|e\rangle$ sets the detection wavelength and also the dark current. (b) Schematics of the possible levels of a quantum well infrared photodetector operating in strong coupling. The dark states (dashed grey line) do not couple with the EM field. They are in the same position as the $|e\rangle$ state in weak coupling and they set the dark current, via thermal activation of the carriers into the quasi-continuum of states $\left(\mathrm{E}_{\mathrm{act}}\right)$. The UP and LP polaritons are instead bosonic excitations, and the level alignment we hypothesize is sketched in the picture. On one hand, detection wavelengths and $E_{a c t}$ are now disentangled. On the other hand, however, we expect a strong suppression of the photocurrent signal originating from the LP.

the detector in the strong light-matter coupling regime [see Fig. 1(b)]. In this case, the thermally activated processes would be dominated by the dark states [dashed grey line in Fig. 1(b)], but the system would instead absorb photons at the polaritonic energies, which are different by $\pm \hbar \Omega_{\text {Rabi }}$ (the Rabi energy). As a consequence, in strong coupling, the thermal activation energy is disentangled from the detection wavelength, and a judicious design could permit one to engineer this difference in favor of a $\mathrm{T}_{\mathrm{BLIP}}$ increase.

It is apparent that all this reasoning naturally raises the question of the polaritonic level alignment with respect to the single particle states. Since the dark states do not couple with the EM field, their energy corresponds to the $|\mathrm{g}\rangle$ and $|\mathrm{e}\rangle$ single-particle states in the QW and their energy is well defined. On the other hand, the energy position of the upper polariton (UP) and lower polariton (LP) with respect to the single-particle energy levels is a more subtle issue as they are mixed bosonic excitations, resulting from a superposition of a photon and a matter excitation. An intuitive reasoning, taking the initial state with all electrons in the $|\mathrm{g}\rangle$ state as the reference, suggests that the correct level alignment is the one sketched in Fig. 1(b). Although various theoretical works exploited this assumption, showing how resonant electron transport can dramatically modify both the efficiency ${ }^{6}$ and the spectral features ${ }^{8,9}$ of polaritonic electroluminescence, no explicit experimental verification of this assumption was so far available. Well beyond inorganic QWs, experiments putting this matter on firm grounds may be of interest for a variety of systems, in particular, organic materials where strong light-matter coupling is responsible for peculiar chemical ${ }^{11-13}$ and transport ${ }^{14-16}$ properties.

In this letter, we demonstrate that quantum well mid-infrared photo-detectors operating in the strong light-matter coupling regime $^{23,24}$ are a system that permits one to build an explicit connection between the energy of the single-particle electronic states and the one of the polaritonic excitations. A relatively highly doped QWIP has been designed at $\approx 9.2 \mu \mathrm{m}$ wavelength. It operates in weak coupling when processed in a mesa configuration and in strong-coupling when featuring a metal/active-region (AR)/grating resonator as in Ref. 17. The comparison between absorption and photocurrent in weak vs strong coupling, supported by a theoretical model, yields information on the level alignment of the polaritonic modes vs single-particle states. By looking at the positions of the polaritonic peaks in absorption vs photo-current, we have been able to confirm the accuracy of the level alignment scheme of Fig. 1(b).

The structure (HM4065) has been grown by molecular beam epitaxy on a SI-GaAs substrate with a 400 -nm-thick stop-layer. It consists of 31 repetitions of 6.5-nm-thick GaAs QWs separated by 20-nm-thick $\mathrm{Al}_{0.25} \mathrm{Ga}_{0.75}$ As barriers. The QWs are nominally doped in the center to $\mathrm{n}_{2 \mathrm{D}}=8.5 \times 10^{11} \mathrm{~cm}^{-2}$, and the structure is sandwiched by 100 -nm-thick $\mathrm{n}^{+}$-GaAs contact layers.

The sample was first characterized by multi-pass waveguide transmission at $300 \mathrm{~K}$ and $78 \mathrm{~K}$. The results are reported in Fig. 2(a). A clear resonant ISB transition at $1098 \mathrm{~cm}^{-1}$ with a FWHM of $\approx 100 \mathrm{~cm}^{-1}$ is measured at $78 \mathrm{~K}$, which we assign to the $|g\rangle \rightarrow|e\rangle$ transition. The thickness of the QW was chosen in a way that the excited $|e\rangle$ state is only marginally bound and its energy lies just below the edge of the confining potential. The "noise" in the region starting at $\approx 1300 \mathrm{~cm}^{-1}$ is a remnant of water vapor absorption.
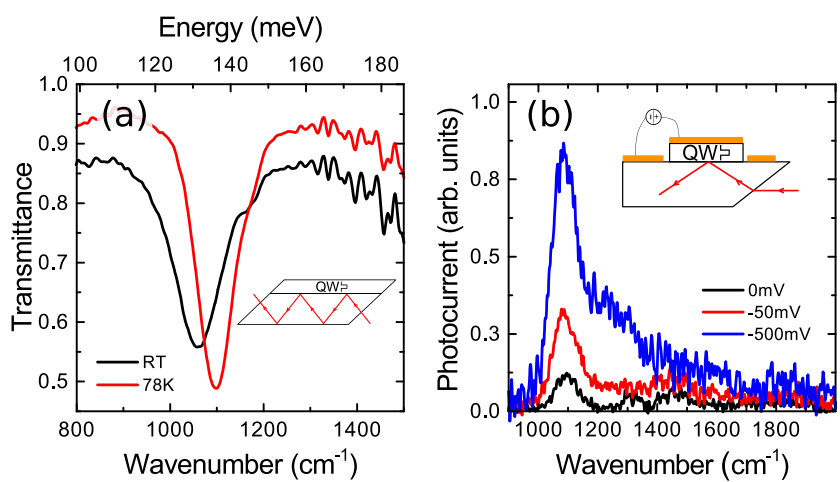

FIG. 2. (a) Transmission spectra (resolution $8 \mathrm{~cm}^{-1}$ ) at $300 \mathrm{~K}$ and $78 \mathrm{~K}$ of the multiple quantum well structure shaped in $45^{\circ}$ multi-pass waveguide configuration. (b) Photocurrent spectra of the QWIP detector in weak coupling at $78 \mathrm{~K}$. The sample was processed as $100-\mu \mathrm{m}$ diameter circular mesas with top and bottom ohmic contacts. 
The samples were then fabricated into photo-detectors as mesa devices with variable sizes as schematically shown in the inset of Fig. 2(b). Both bottom and top contacts are ohmic: the former one is based on $\mathrm{Ni} / \mathrm{Ge} / \mathrm{Au}$, while the latter one on $\mathrm{Pd} / \mathrm{Ge}$. The samples were then polished to obtain a $45^{\circ}$ facet and mounted on a copper block for cryogenic measurements. Typical photocurrent spectra at $78 \mathrm{~K}$ are shown in Fig. 2(b) for different applied biases. A resonant, TMpolarized response at $1085 \mathrm{~cm}^{-1}$, with a FWHM of $100 \mathrm{~cm}^{-1}$ is observed that we assign to the $|g\rangle \rightarrow|e\rangle$ transition. A high energy shoulder appears at high bias: it can be explained by the transitions into the quasi-continuum of states above the barrier.

The mesa device operates in weak light-matter coupling as there is no bottom mirror and no well-defined resonant cavity mode, but just a continuum of propagating leaky modes. The same AR can be operated in strong coupling if a bottom mirror is employed, i.e., if it is inserted in a metal-AR-metal waveguide. As it is discussed in full detail in Refs. 18 and 19, this yields a pair of polaritonic modes with a sizable Rabi splitting $2 \Omega_{\text {Rabi }}=\left(\omega_{U P}-\omega_{L P}\right)=\sqrt{f_{w}} \omega_{\text {plasma }}$, where $f_{w}$ is the fraction of the optical mode overlapping with the active QWs and $\omega_{\text {plasma }}$ is the plasma frequency in the QWs. We have first implemented a very large $\left(4 \times 4 \mathrm{~mm}^{2}\right)$ metal-AR-grating passive dispersive resonator with sample HM4065 using the design and fabrication procedure reported in Ref. 17. This architecture is extremely practical as the polaritonic dispersion can be easily probed with angular-resolved surface reflectivity. Figure 3(a) shows the room-temperature photonic dispersion (energy vs incidence angle, color plot) $R(E, \theta)$ as inferred from the reflectivity measurements. The upper (UP) and lower (LP) polaritonic branches are clearly observed, evidence that the system operates in the strong light-matter coupling regime.

The dots in Fig. 3(a) indicate the position of the reflection minima predicted for the two polaritonic branches using a RCWA ${ }^{19}$ (Rigorous Coupled Wave Analysis) numerical simulation of the structure with the nominal parameters, except for the doping that has been set to $n_{2 \mathrm{D}}$ $=5.5 \times 10^{11} \mathrm{~cm}^{-2}$, instead of $8.48 \times 10^{11} \mathrm{~cm}^{-2}$ as per growth sheet. Once this reduced doping level is included, the agreement with the experiment turns out to be excellent, which fully confirms that RCWA simulations can be employed an extremely predictive numerical tool in the analysis that follows.

To implement a detector using this architecture, proper electrical contacts must be added. ${ }^{20}$ A sketch of a typical device is presented in the inset of Fig. 3(b), with the details described in the caption. Since the information on the alignment of the polaritonic modes vs. singleparticle states is obtained from the comparison between reflectivity and photocurrent spectra, it is crucial to verify that the polaritonic reflectivity (i.e., absorption) is not affected by the presence of the electric field when the detector is operated under bias. Figure 3(b) shows reflectivity spectra at $78 \mathrm{~K}$ for different biases performed on a $3.5-\mu \mathrm{m}$ grating period, $80 \%$ filling factor (ff) SC-QWIP device. The overall size of the grating $\left(150 \times 150 \mu \mathrm{m}^{2}\right)$ is much smaller than the one of the passive devices, so reflectivity measurements have been performed with a FTIR microscope at a fixed incidence angle of $20^{\circ} \pm 5^{\circ}$. The results clearly confirm that the polaritonic absorption is totally unaffected by the applied bias.

As a next step, we have measured the spectral response of the photocurrent for three QWIP devices cooled at liquid nitrogen temperatures, for different angles of incidence, using an FTIR with a Globar source. The results are reported in Figs. 4(a), 4(b), and 4(c) as
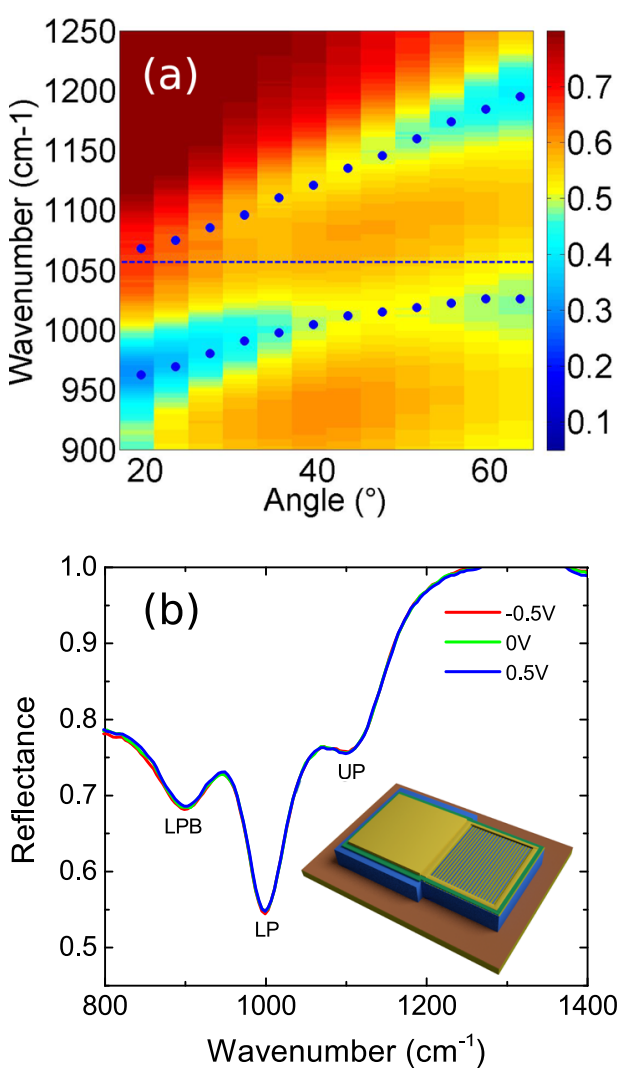

FIG. 3. (a) Room temperature reflectivity dispersion from $17^{\circ}$ to $65^{\circ}$ of a $2.5 \times$ $2.5 \mathrm{~mm}^{2}$ polaritonic cavity. The grating period is $3.8 \mu \mathrm{m}$ and the filling factor is $70 \%$. The blue dots correspond to the RCWA simulation with an ISB transition at 31.7 $\mathrm{THz}$ and a doping of $5.5 \times 10^{11} \mathrm{~cm}^{-2}$. (b) Reflectivity spectra at $20^{\circ} \pm 5^{\circ}$ and $78 \mathrm{~K}$ acquired with a FTIR microscope (resolution is $8 \mathrm{~cm}^{-1}$ ) on a $150 \times 150 \mu \mathrm{m}^{2}$, $3.5 \mu \mathrm{m}$ period and $80 \%$ filling factor SC-QWIP under different biases. The upper and lower polaritonic branches are indicated as UP and LP, while LPB indicates the Bragg-folded lower photonic branch. ${ }^{17}$ There is no change in absorption as a function of the applied voltage. The sample temperature is $78 \mathrm{~K}$. The inset shows the schematic of a SC-QWIP device: active region (blue), SiN insulator (green), gold top contact (yellow) and ohmic PdGe bottom contact (dark orange).

color plots, where each photocurrent spectrum at a given angle has been normalized to have a maximum value of 1 (the spectra for each angle are reported in the supplementary material). The three devices differ for the grating period $(4 \mu \mathrm{m}, 3.7 \mu \mathrm{m}$, and $3.2 \mu \mathrm{m})$. For each of them, the polaritonic dispersion has been calculated using RCWA simulations and is represented on the color plots by the white dots. No adjustable parameters are used, and the real grating dimensions-as obtained from the SEM images-are employed in the simulations. Since the very small $\left(150 \times 150 \mu \mathrm{m}^{2}\right)$ size of the detector devices made it extremely challenging to directly measure the angle-resolved reflectivity and extract the polaritonic dispersion, we had to rely on RCWA simulations that - as demonstrated above-are highly accurate.

The color plots of the photocurrent response reveal that the SCQWIP detectors do operate in the strong light-matter coupling regime: in contrast to the single peaked response found in the weak coupling shown in Fig. 2(b) [white dashed line in Figs. 4(a), 4(b), and 4(c)], two 


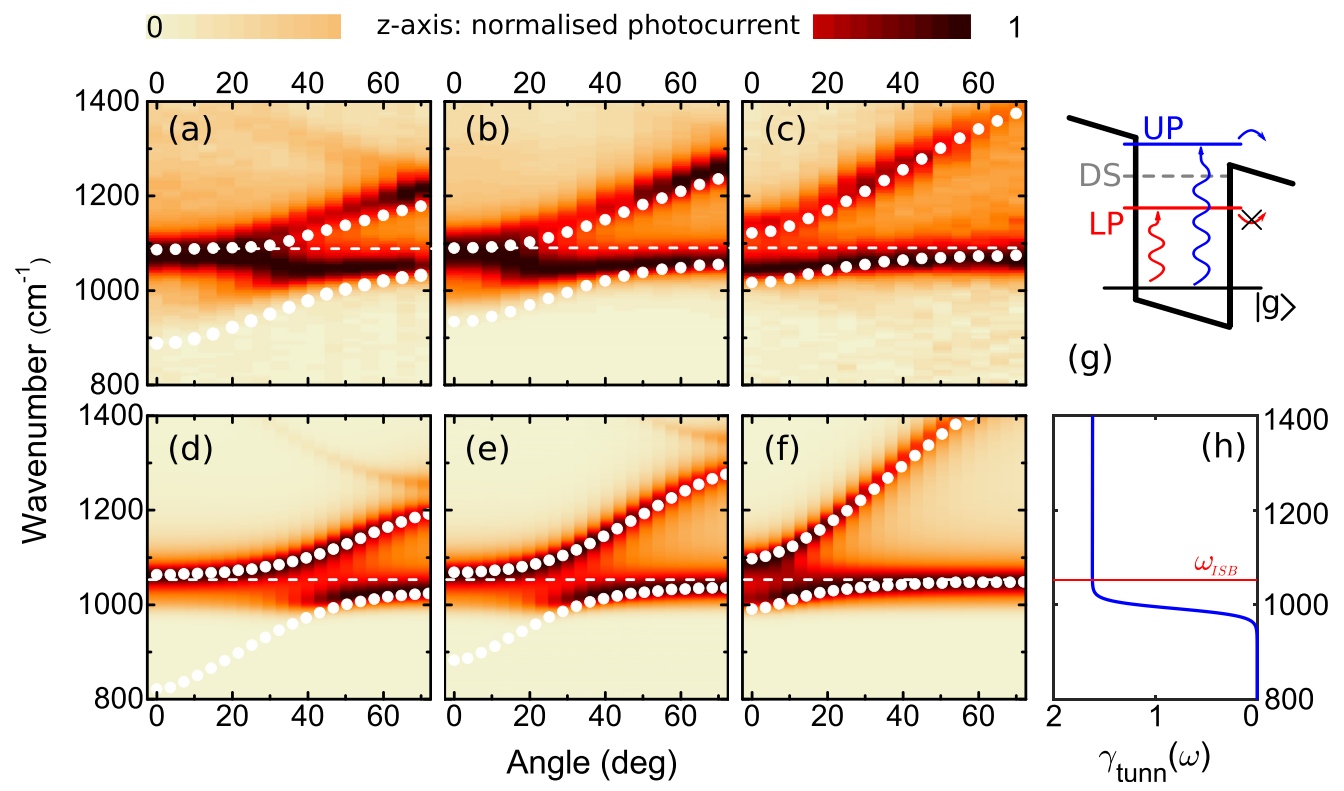

FIG. 4. (a)-(c) Angle-resolved spectral photocurrent for three SC-QWIPs (150 $\mu \mathrm{m}$ side, $80^{\circ}$ filling factor) with grating periods of $4 \mu \mathrm{m}, 3.7 \mu \mathrm{m}$, and $3.2 \mu \mathrm{m}$ in panels (a), (b), and (c), respectively. The measurements are performed at $78 \mathrm{~K}$, with a resolution of $12 \mathrm{~cm}^{-1}$, for an angle span from $0^{\circ}$ to $70^{\circ}$. The applied bias are $450 \mathrm{mV}, 500 \mathrm{mV}$ and $700 \mathrm{mV}$, respectively, for the measurements in panels $a, b$, and $c$. The white dots correspond to the $2 \mathrm{D}$ RCWA simulation for each device, with the following same parameters (except for the grating): top metal thickness $200 \mathrm{~nm}$; filling factor $80 \%$; active region thickness $940 \mathrm{~nm}$; bottom metal $400 \mathrm{~nm}$; ISB transition $32.8 \mathrm{THz}$; doping $5.5 \times 10^{11} \mathrm{~cm}^{-2}$. The spectra for each angle and each grating (as measured and normalized) are reported in the supplementary material. (d) $-(f)$ Theoretical color plots of the dependence of the photocurrent intensity on the frequency and angle of the incident light. The three panels correspond to the three devices used for the experimental plots in Fig. 3. The details of the model are given in Ref. 13. Parameters used in the calculations: $\Omega_{\text {Rabi }}=57 \mathrm{~cm}^{-1}, \gamma_{\mathrm{nr}}=32 \mathrm{~cm}^{-1}, \gamma_{\mathrm{rad}}=20 \mathrm{~cm}^{-1}, \gamma_{\mathrm{hom}}=65 \mathrm{~cm}^{-1}$, and $\omega_{\text {ISB }}=1053 \mathrm{~cm}^{-1}$. The photocurrent transfer function is plotted in Fig. 4. Other cavity parameters: effective refractive index $n_{0}=3.1$, Bragg frequency $\omega_{\text {Bragg }}=24 \mathrm{~cm}^{-1}$, grating periods a $=$ $4.0 \mu \mathrm{m}$ (panel d), $3.7 \mu \mathrm{m}$ (panel e), $3.2 \mu \mathrm{m}$ (panel f). (g) Schematics of the levels of a quantum well infrared photo-detector operating in strong coupling that explains the data in panels $(\mathrm{a})-(\mathrm{c})$. (h) Model photocurrent transfer function $\gamma_{\text {tunn }}(\omega)$ used in the calculations of panels $(\mathrm{d})-(\mathrm{f})$.

dispersive branches clearly appear, which we attribute to polaritons. Their sizable Rabi splitting $2 \Omega_{\text {Rabi }}$ allows our SC-QWIP to cover a broad range of wavelengths from 10 to $7.2 \mu \mathrm{m}$ with a relatively flat efficiency by using a given electronic resonance around $9 \mu \mathrm{m}$ in the AR and to display a wide frequency selectivity simply by tuning the angle. While the UP branch in detection closely follows the polaritonic dispersion extracted from the reflectivity dips (white dots) or, equivalently, from absorption peaks, the LP branch displays an interesting feature, as it does not extend to wavelengths much longer than the weak-coupling resonance and the strength of its lower part quickly fades away.

This interesting finding can be understood in terms of the level alignment sketched in Fig. 1(b) and reported in Fig. 4(g) for clarity. Taking the initial state with all electrons in the ground state of the well as a reference for energies, the UP positions itself at higher energy with respect to the QW edge. As a result, it overlaps with the electronic states in the external quasi-continuum, so we can expect that the electron promoted to the excited state is able to escape the QW and give rise to a sizable photocurrent signal. On the other hand, the relatively large value of the Rabi splitting $2 \Omega_{\text {Rabi }}$ pushes the energy of the LP below the bare $g \rightarrow e$ transition energy and, thus, well below the QW edge. As a result, the electron does not have enough energy to escape above the QW edge and the potential barrier for the moderate values of the applied electric field in the $1-5 \mathrm{kV} / \mathrm{cm}$ range is too large to allow a significant tunneling. The photocurrent is therefore strongly suppressed.

While this qualitative picture is already quite clear, extracting quantitative information from the data requires a theoretical model of the photocurrent process. Here, we will focus on a simple phenomenological model that is able to accurately reproduce the experimental data in terms of a single transfer function summarizing the frequency dependence of the probability rate of escape from the QW for an electron in the excited $(e)$ state. This process is included in the temporal coupled mode theory of laterally patterned cavities in strong lightmatter coupling regime developed in Ref. 21. The escape of electrons from the QW is included in the theory as an additional, frequencydependent decay of the ISB transition of rate $\gamma_{t u n n}(\omega)$.

In its simplified formulation for spatially homogeneous cavities (the complete case is straightforwardly built along the lines of Ref. 21), the steady-state of the cavity photon $a_{k}$ and ISB $b_{k}$ fields under a monochromatic and spatially plane-wave incident electromagnetic field of amplitude $E_{i n c}$ and in-plane wavevector $\boldsymbol{k}$ at frequency $\omega$ reads

$$
\begin{gathered}
\omega a_{k}=\omega_{c a v, k} a_{k}-i\left(\gamma_{\text {rad }}+\gamma_{n r}\right) a_{k} / 2+\Omega_{\text {Rabi }} b_{k}+E_{\text {inc }}, \\
\omega b_{k}=\omega_{I S B} b_{k}+\Omega_{\text {Rabi }} a_{k}-i\left[\gamma_{\text {hom }}+\gamma_{\text {tunn }}(\omega)\right] b_{k} / 2,
\end{gathered}
$$

where $\omega^{c a v}{ }_{k}$ is the photon dispersion along the cavity plane, the effective frequency $\omega^{I S B}$ of the bright ISB transition coupled to the cavity 
mode includes the depolarization shift, ${ }^{15} \Omega_{R a b i}$ is the Rabi frequency of the light-matter coupling, and $\gamma_{\text {rad }}, \gamma_{n r}$ and $\gamma_{I S B}$ are, respectively, the radiative and non-radiative loss rate of the cavity photon and the homogeneous decay rate of the ISB transition. In this formalism, the photo-current intensity is modulo a constant factor

$$
\mathrm{I}_{\mathrm{pc}}=\gamma_{\text {tunn }}(\omega)\left|b_{\boldsymbol{k}}\right|^{2} .
$$

On physical grounds, we can anticipate that a reasonable choice for $\gamma_{\text {tunn }}(\omega)$ is to have a sharp cut-off on the low-energy side slightly below the effective ISB transition frequency $\omega_{I S B}$ and a slow decay at high frequencies. As it is sketched in Fig. 4(g), escape from the QW is rapidly suppressed when the electron energy is pushed well below the QW edge (following a Fowler-Nordheim behavior), while the highfrequency decay is due to the reduced overlap between the electron wavefunction in the QW and the one of fast moving outgoing states.

An example of such a shape is shown in Fig. 4(h) under the simplifying assumption that the electron tunneling decays very slowly at high frequencies. The corresponding color plots of the frequencyand angle-dependent photocurrent spectrum are shown in Figs. 4(d) -4 (f) for the three values of lattice spacing used in the experiments. White dots indicate the position of the reflectivity minima calculated using the same theory. As one can see, upon comparing these plots with the experimental ones shown in Figs. 4(a)-4(c), the agreement on the position of the reflection minima and of the photocurrent maxima is excellent. Even more importantly, the dramatic disappearance of the lower photonic branch at low energies is accurately reproduced by the theory.

While the simple transfer function $\gamma_{\text {tunn }}(\omega)$ that we have employed qualitatively validates the level alignment proposed in Fig. 1(b), it is important to note that agreement between the theory and the experiment requires that the low-energy cut-off of the tunneling rate $\gamma_{\text {tunn }}(\omega)$ be located at $\delta_{\text {cut }} \approx 50 \mathrm{~cm}^{-1}$ below the ISB transition frequency $\omega^{I S B}$, as displayed in Fig. 4(h). Even though a more sophisticated theory is required to firmly assess the origin of this shift, a tentative explanation involves the depolarization shift due to Coulomb interactions in the QW, which blue-shifts the effective ISB frequency $\omega_{I S B}$ (and thus the horizontal asymptote of the LP) above the singleparticle energy difference $\omega_{\text {eg }}=\left(\omega_{\mathrm{e}}-\omega_{\mathrm{g}}\right)$ (which is by design located just below the QW edge). Using the real doping level of the current sample, we obtain in fact a value for the depolarization shift of $\approx 41 \mathrm{~cm}^{-1}$, roughly comparable in magnitude to $\delta_{\text {cut }}$. This simple estimation thus reinvigorates the simple physical picture that tunneling is quickly suppressed as the energy of the incident photon drops below the QW edge. The development of a microscopic theory of the electronic transport in a QWIP detector able to quantitatively predict the relative intensities of the polaritonic photocurrent features will be the immediate follow-up of this work.

In summary, we have reported an experimental study of the photo-current signal of a QWIP whose active region is strongly coupled to a cavity photon mode. The photo-current is peaked on the polaritonic branches, the lowest part of the lower polariton being suppressed by the suppressed electron escape at low energy. This observation not only offers quantitative information on the relative energy alignment of fermionic electronic states in the QW and of bosonic polaritonic modes but also suggests QWIPs as a powerful tool to approach urgent fundamental questions arising in the entire polaritonic community, for both inorganic QW-based ${ }^{22}$ and organic systems. ${ }^{10,13}$ From the applicative point of view, our results highlight the potential of SC-QWIPs to allow angle-dependent frequency tuning of the detection wavelength of a given electronic structure. Finally, such results can also open interesting perspectives on both the physics and the optimization of many other devices such as polaritonic QCDs or polaritonic LEDs.

See supplementary material for photodetection spectra of the SCQWIPs. The data are presented as measured and also normalized.

We thank Simone De Liberato, Alexandre Delga, Jean-Jacques Greffet, and Francois Julien for useful discussions. We acknowledge financial support from the European Union FET-Open Grant MIRBOSE (737017) and the European Research Council (IDEASERC) ("GEM") (306661). This work was partly supported by the French RENATECH network and Provincia Autonoma di Trento.

\section{REFERENCES}

${ }^{1} \mathrm{H}$. (Harald) Schneider and H. C. Liu, Quantum Well Infrared Photodetectors: Physics and Applications (Springer, 2007).

${ }^{2}$ D. Palaferri, Y. Todorov, A. Bigioli, A. Mottaghizadeh, D. Gacemi, A. Calabrese, A. Vasanelli, L. Li, A. G. Davies, E. H. Linfield, F. Kapsalidis, M. Beck, J. Faist, and C. Sirtori, Nature 556, 85 (2018).

${ }^{3}$ S. De Liberato and C. Ciuti, Phys. Rev. Lett. 102, 136403 (2009).

${ }^{4}$ P. Jouy, A. Vasanelli, Y. Todorov, L. Sapienza, R. Colombelli, U. Gennser, and C. Sirtori, Phys. Rev. B 82, 45322 (2010).

${ }^{5}$ R. Colombelli and J.-M. Manceau, Phys. Rev. X 5, 011031 (2015).

${ }^{6}$ S. De Liberato and C. Ciuti, Phys. Rev. B: Condens. Matter Mater. Phys. 79, 75317 (2009).

${ }^{7}$ C. F. Bohren and D. R. Huffman, Absorption and Scattering of Light by Small Particles (Wiley, 1998).

${ }^{8}$ M. Cirio, S. De Liberato, N. Lambert, and F. Nori, Phys. Rev. Lett. 116, 113601 (2016).

${ }^{9}$ M. Cirio, N. Shammah, N. Lambert, S. De Liberato, and F. Nori, e-print arXiv:1811.08682 (2018).

${ }^{10}$ A. Thomas, J. George, A. Shalabney, M. Dryzhakov, S. J. Varma, J. Moran, T. Chervy, X. Zhong, E. Devaux, C. Genet, J. A. Hutchison, and T. W. Ebbesen, Angew. Chem., Int. Ed. 55, 11462 (2016).

"J. Galego, F. J. Garcia-Vidal, and J. Feist, Phys. Rev. X 5, 041022 (2015).

${ }^{12}$ J. Flick, M. Ruggenthaler, H. Appel, and A. Rubio, Proc. Natl. Acad. Sci. 114, 3026 (2017).

${ }^{13}$ E. Orgiu, J. George, J. A. Hutchison, E. Devaux, J. F. Dayen, B. Doudin, F. Stellacci, C. Genet, J. Schachenmayer, C. Genes, G. Pupillo, P. Samorì, and T. W. Ebbesen, Nat. Mater. 14, 1123 (2015).

${ }^{14}$ G. L. Paravicini-Bagliani, F. Appugliese, E. Richter, F. Valmorra, J. Keller, M. Beck, N. Bartolo, C. Rössler, T. Ihn, K. Ensslin, C. Ciuti, G. Scalari, and J. Faist, Nat. Phys. 15, 186 (2019).

${ }^{15}$ D. Hagenmüller, J. Schachenmayer, S. Schütz, C. Genes, and G. Pupillo, Phys. Rev. Lett. 119, 223601 (2017).

${ }^{16}$ J.-M. Manceau, S. Zanotto, T. Ongarello, L. Sorba, A. Tredicucci, G. Biasiol, and R. Colombelli, Appl. Phys. Lett. 105, 81105 (2014).

${ }^{17}$ F. Alpeggiani and L. C. Andreani, Phys. Rev. B 90, 115311 (2014).

${ }^{18}$ Y. Todorov, Phys. Rev. B 89, 75115 (2014).

${ }^{19}$ J. M. Manceau, S. Zanotto, I. Sagnes, G. Beaudoin, and R. Colombelli, Appl. Phys. Lett. 103, 91110 (2013).

${ }^{20}$ D. Chastanet, J.-M. Manceau, T. Laurent, A. Bousseksou, G. Beaudoin, I. Sagnes, and R. Colombelli, Appl. Phys. Lett. 110, 81108 (2017).

${ }^{21}$ J. M. Manceau, G. Biasiol, N. L. Tran, I. Carusotto, and R. Colombelli, Phys. Rev. B 96, 235301 (2017).

${ }^{22}$ E. Cortese, I. Carusotto, R. Colombelli, and S. De Liberato, Optica 6, 354-361 (2019).

${ }^{23}$ L. Sapienza, A. Vasanelli, C. Ciuti, C. Manquest, C. Sirtori, R. Colombelli, and U. Gennser, Appl. Phys. Lett. 90, 201101 (2007).

${ }^{24}$ E. Dupont, H. C. Liu, A. J. SpringThorpe, W. Lai, and M. Extavour, Phys. Rev. B 68, 245320 (2003) 